\section{Growing with the Grain}

Though it derives inspiration from the Grameen Bank, which makes loans to poor women in emerging economies, Gramene takes a decidedly different approach to improving the world, by trading in the currency of information, not money. The site, which is designed as a resource for comparative grass genomics, provides a cornucopia of research data on rice, sorghum, corn, wheat, barley, oats, and millet. Visitors can browse assembled genomes, search for protein structures, compare genetic maps, find genetic markers, examine phenotype-related loci, scan metabolic pathways, browse relevant literature, and upload information. They can also stay on top of news in the field, and there are even tutorials for newbies. That's a breakfast of champions for researchers and a relatively easy way to stay on top of this rapidly changing "landscape."

\section{www.gramene.org}

\section{Epigenome Home}

We think of phenotypes as manifestations of nature and nurture, but the more the molecular underpinnings of biology are determined, the more there is to marvel about their complexity. For example, it is apparent that there is more to the nature component than genotype. Witness the epigenomic phenomena of imprinting, gene silencing, $\mathrm{X}$ chromosome inactivation, position effects, and bookmarking, which can all vary in an organism, despite the fact that the cells have identical DNA sequences. Experience the excitement of this rapidly evolving field at the Epigenome Network of Excellence.

\section{www.epigenome-noe.net}

\section{Pro-Creative}

When was the last time you were asked to pontificate or "commentate"? If you're like most of us, you probably can't remember, but this can be changed quickly on your first visit to Science Creative Quarterly (SCQ), where scientists' opinions and literary talents not only matter, but are encouraged. With a format aimed at bringing out researchers' "inner voices," David Ng's SCQ provides online opportunities to expand the creative horizons of its visitors. Looking for a laugh? You'll enjoy the humor section, which is peppered with funny articles

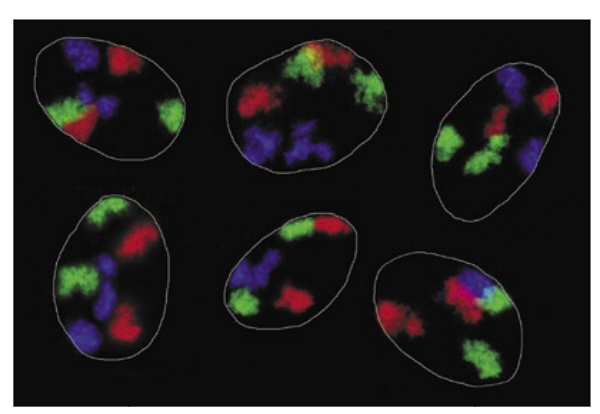

C2005 http://www.epigenome-noe.net/researchtools/protocol.php?protid=23

like "Glacier Restoration Project Creeps Forward" or "Miracle on the 34th Replicate." Serving a large spectrum spanning nerdy but talented writers to hungry readers, SCQ is indeed a rare medium, well done.

\section{www.scq.ubc.ca}

\section{Fume-Free Fun}

People who like chemistry but can't stand to wear goggles and lab jackets don't have many options in pursuing their passion. They can ( $i$ ) abandon the subject altogether or (ii) work in a virtual lab. The latter has several advantages, including numerous opportunities to learn without getting one's hands dirty and at virtually no expense, since the costs of chemicals, waste disposal, and the hazards of exposure are all virtual, too. If such a setup is your cup of tea, you should check out the ChemCollective. Noteworthy for its strong educational content, a pricing scheme that fits into any budget, and the complete absence of chemicals, the site works for both students and faculty, with content that includes not only clever lab exercises, but also tutorials for students and activities for instructors. There's even a mystery to solve.

\section{www.chemcollective.org}

\section{Flocks of LOX}

When it comes to enzymes linked to discomfort, you'll have to look pretty hard to find a more troublesome set than the lipoxygenases (LOX). The catalysts for forming leukotrienes and related compounds from arachidonic acid in animals (and linolenic acid in plants), lipoxygenases produce molecules implicated in inflammation, asthma, arthritis, and cancer. Ouch! Through knowledge comes power, though, and the LipOXygenases DataBase (LOX-DB) is a significant force, empowering researchers and helping them tackle these problems. It works by providing tools common to modern molecular research, including aligned sequences, ribbon diagrams, BLASTing, and sequence comparison matrices. A searchable reference section and another describing the relationship between LOX products and cancer round out this interesting site.

\section{www.dkfz.de/spec/lox-db/}

\section{-Kevin Ahern - Please send web site} recommendations to ahernk@orst.edu 